\title{
Le diagnostic préimplantatoire n'est pas un diagnostic prénatal précoce
}

\author{
Jacques Testart, Bernard Sèle
}

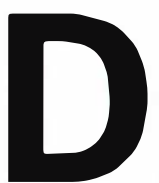
ans leur article en faveur du diagnostic préimplantatoire (DPI), Viville et al. [1] critiquent notre opposition à cette méthode, telle que nous l'avons exprimée depuis plusieurs années [2,3].

D'abord, évitons les faux débats. Nous n'avons jamais imaginé que le DPI puisse être capable de sélectionner "l'enfant parfait" et c'est heureux; il n'est donc pas honnête de réduire notre argumentation à l'expression d'un fantasme: "puisque c'est de cela qu'il s'agit, le surhomme des fantasmes souvent exprimés dans la littérature et le cinéma" [1]. Nous n'ignorons pas que toute personne dite "normale" porte non seulement plusieurs gènes caractéristiques de graves maladies mais aussi d'innombrables autres facteurs de risque, et que cet état "naturel " résistera toujours aux rêves de santé permanente, et d'immortalité. C'est d'ailleurs pourquoi nous pensons que, malgré les exploits remarquables des généticiens, le DPI apportera davantage à l'idéologie normative qu'à la normalisation de l'espèce.

Autre argument fallacieux, celui qui proclame "assez dérisoire" l'opposition au DPI sous prétexte que "l'avortement pour convenance personnelle est bien accepté dans notre société». En réalité, nous ne souhaitons pas sacraliser l'embryon mais faire remarquer que la sélection du "meilleur", embryon, conduit à instrumentaliser l'homme (pas seulement au stade larvaire mais comme identité à l'enchaînement des bases constituant son ADN. En effet, conjointement à la sélection médicale pourra intervenir une sélection de convenance personnelle inspirée par les considérations idéologiques du moment.

Selon les auteurs, qui l'affirment à deux reprises, le DPI «est à considérer comme une forme de diagnostic prénatal précoce". C'est ce que nous contestons formellement. Quand ils reconnaissent que le DPI «est clairement plus facile à accepter qu'une IMG", ils contredisent l'affirmation qu'entre l'un et l'autre il n'y aurait qu'une nuance de calendrier. Quelle portée accorder alors à la règle énoncée que "dans un premier temps seules les maladies d'une particulière gravité, incurables et touchant le jeune enfant doivent être envisagées " ? S'il ne devait pas y avoir de second temps ouvrant des perspectives de moindre exigence nous pourrions nous aussi applaudir au DPI. Les généticiens n'auraient-ils pas assez confiance dans leur discipline qu'ils se montrent tellement dubitatifs quant aux progrès de leurs techniques? Car à mesure que s'améliorera la reproductibilité, la fiabilité, la polyvalence des tests, en même temps que leur coût diminuera et que les techniques d'AMP (assistance médicale à la procréation) se feront plus efficaces, que deviendront les indications médicales du DPI? D'ailleurs dès à présent, la réalisation de DPI pour risque d'hémophilie [4], d'accident rhésus [5] ou de polypose colique familiale [6] est le signe avant coureur d'une dérive des indications habituelles du DPN. Aussi est-il inexact d'affirmer que «le DPI est proposé, actuellement, uniquement pour des maladies monogéniques graves, sans espoir pour l'enfant à naître..." [1].

De plus, la proposition de thérapie génique germinale pour prévenir d'autres affections comme le cancer du sein [7], ne peut qu'évoluer vers le DPI dès que l'absurdité du projet sera révélée : pourquoi «soigner» un embryon malade alors qu'il existe des embryons sains en surnombre dans la même éprouvette? Si l'on ne peut que partager le souci d'alléger la souffrance occasionnée par l'IMG, doit-on pour autant ignorer, que la proposition du DPI sera infiniment plus lourde de conséquence au plan collectif que l'alternative du DPN auquel elle permettra d'échapper au plan individuel.

Le Tableau I illustre l'impact des diverses pratiques eugéniques selon la cible choisie. Il apparaît que le DPI, dernière venue de ces pratiques, est la plus acceptable par la société comme par le couple impliqué. De plus, cette technique est la seule susceptible d'un réel effet eugénique. Face à des possibilités de tri embryonnaire qui ne peuvent aller qu'en s'accroissant, le désir d'enfant idéal, même illusoire, ne peut en aucune façon régresser. Ce désir, inscrit depuis le début des temps dans les mythes parentaux, s'intègre de plus en plus dans la responsabilité de ceux qu'on désigne aujourd'hui comme "géniteurs". La vraie question est donc de savoir quelles bar- 
Tableau I

AVANTAGES (+) ET INCONVÉNIENTS (-) DES DIVERSES PRATIQUES EUGÉNIQUES EN FONCTION DE LEUR CIBLE RESPECTIVE

\begin{tabular}{|lccc|}
\hline $\begin{array}{l}\text { Cible de } \\
\text { l'action eugénique }\end{array}$ & $\begin{array}{c}\text { Acceptabilité } \\
\text { sociale }\end{array}$ & $\begin{array}{c}\text { Satisfaction } \\
\text { du couple }\end{array}$ & $\begin{array}{c}\text { Conséquences } \\
\text { eugéniques }\end{array}$ \\
\hline Adulte & & & 0 \\
$\quad$ - stérilisation & \pm & - & 0 \\
$\quad$ conseil génétique & + & - & 0 \\
Nouveau-né (infanticide) & - & \pm & 0 \\
Fœtus (IMG) & \pm & ++ & + \\
Embryon (non transfert & + & & \\
in utero) & & & \\
\hline
\end{tabular}

rières sont susceptibles d'être élevées entre une demande illimitée et des offres sans limites.

Espérer un autocontrôle des patients ou du corps médical est aussi naif que d'imaginer les citoyens acquittant leurs impôts sans y être obligés; la «qualité" d'un enfant suscite trop d'intérêts, objectifs ou imaginés, pour que l'organisation sociale accepte de s'en remettre à une telle régulation spontanée. Comment ne pas apercevoir le pouvoir conféré aux généticiens, dès lors que le principe du tri des embryons aura été admis?

$\mathrm{Ou}$ alors les propagateurs du DPI estiment, comme souligné fortement par certains [8], qu'il appartient aux seuls couples de définir le niveau d'exigence génétique souhaité pour leurs enfants. Or, on connaît la conformation des désirs individuels à des modèles collectifs. Et si le seul désir parental doit faire loi, pourquoi s'opposer à d'autres possibilités offertes par la procréation assistée (clonage, parthénogenèse, etc.) ?

Viville et al. écrivent que notre point de vue critique "a fait vivement réagir la communauté scientifique internationale». Si l'expression est un peu forte pour qualifier une poignée de généticiens impliqués dans le tri embryonnaire, il reste que des arguments nous ont été opposés auxquels il faut répondre.

Bien que nous n'ayions pas imaginé que le DPI irait jusqu'à concerner les “75000 à 100000 gènes différents que possède notre génome", nous ne comprenons pas pourquoi Viville et al. limitent à 1 ou 2 le nombre des cellules embryonnaires sur lesquelles les diagnostics peuvent être réalisés. Il est en réalité possible de cultiver des blastomères humains isolés pour les multiplier [9] ou même de constituer de véritables clones de millions de cellules [10] sur lesquelles on pourrait potentiellement réaliser plus de tests qu'il n'en existera jamais. Quant au nombre d'embryons analysables, le Tableau II montre comment la répétition des procédures (FIV + DPI) peut conduire à choisir le "meilleur" parmi des dizaines d'enfants potentiels. Le caractère irrationnel de ce choix ne fait pas de doute si l'on se réfère aux effets bénéfiques de certaines mutations (dont des mutations pathogènes), telle celle du co-récepteur CCR5, qui confère une résistance au VIH. Au-delà, c'est bien la banalisation d'une telle procédure qui est au cœur du débat éthique, car elle met en cause le principe d'altérité et la définition de la personne humaine, au profit d'une aggravation de l'idéologie compétitive. Comme chacun le sait, il est possible de définir des qualités animales propres à satisfaire des besoins humains. Ainsi la sélection patiente des meilleures bêtes au sein de petits troupeaux permet à l'homme d'améliorer à son profit les espèces domestiques. On constate ici que des choix portant sur des individus peuvent avoir des conséquences collectives sur l'espèce. Or, certains refusent l'usage du terme "eugénisme" pour qualifier le tri embryonnaire au motif que le DPI intervient sur des individus et non sur des populations [8]. Outre que le DPI comporte l'analyse collective et simultanée de nombreux enfants potentiels (figure 1), ces auteurs omettent que l'eugénisme des pays démocratiques au début de ce siècle a stérilisé des dizaines de milliers d'individus, un par un, à partir d'informations concernant chaque personne.

Viville et al. ne décèlent l'eugénisme qu'après "éradication de l'ensemble des mutations à l'échelle de la population terrestre " confondant par là une pratique (et une idéologie) avec son résultat. Une telle conception d'un génome universel tout propre et achevé est évidemment illusoire et si l'eugénisme n'était qualifié qu'après avoir réussi la purification intégrale, on devrait reconnaître qu'il n'y eut jamais d'eugénistes... Ce n'est pas l'annonce d'un plan global qui caractérise l'eugénisme, c'est l'application à des individus d'un consensus d'exclusion sur des critères biologiques.

D'autres arguments sont venus de "la communauté scientifique internationale». Ainsi Alan Handyside, pionnier du DPI, nous oppose qu'il serait impossible de sélectionner des embryons pour autre chose que des caractères ordinaires (common traits) puisque seuls 5 ou 6 embryons sont disponibles en moyenne [11]. Encore une fois nous n'accusons pas les partisans du DPI de vouloir construire le surhomme des romans mais nous craignons que cette entreprise ne réduise l'humain aux caractères ordinaires de la "normalité »... Remarquons aussi que, pourvu que le DPI soit fréquemment associé à la FIVETE (fécondation in vitro et transplantation d'embryon), l'enjeu des protocoles de stimulation ovarienne sera d'augmenter encore le nombre des ovules recueillis pour disposer d'embryons plus nombreux.

Ce texte [11], comme d'autres [12], dissimule l'effet d'exclusion du DPI (par élimination des embryons "défectueux") au profit d'un projet d'élection (par recherche du "meilleur" embryon résiduel). En fait le DPI va retenir les moins mauvais embryons et non pas ceux of desirable characteristics (figure 1). Insister sur l'élection, c'est privilégier une attitude positive, comme pour se démarquer des abus de l'eugénisme 


\begin{tabular}{|lccc|}
\hline \multicolumn{4}{|c|}{ Tableau II } \\
EFFICACITÉ RELATIVE DES DIAGNOSTICS PRÉIMPLANTATOIRES (DPI) \\
ET PRENATAL (DPN) : NOMBRE D'ENFANTS POTENTIELS \\
SOUMIS A L'ANALYSE POUR UN COUPLE
\end{tabular}

* Avec prélèvement de villosités choriales ou amniocentèse ou échographie. ** Avec examen génétique des embryons issus de FN. négatif du début de ce siècle; pourtant, l'eugénisme négatif reste par définition celui qui exclut et non celui qui sévit dans la douleur. C'est peut-être pour se démarquer de la violence de l'eugénisme historique, essentiellement négatif, que le DPI est montré sous un aspect "positif", comme si le recours à des pratiques indolores évinçait la question idéologique de la «qualité humaine». En fait, puisqu'il s'agit d'éliminer certains embryons pour simultanément en élire d'autres, le DPI institue un eugénisme négatif et positif à la fois. Et, aucune de ces deux voies ne peut être tenue innocente quant à la définition et au respect de la personne humaine.

La conclusion de l'article d'Handyside [11] mérite d'être analysée audelà de son humour apparent: $M y$ advice to any one wanting a child with desirable characteristics, therefore is to practise eugenics in the time-honoured way and select a mate with those characteristics. After all, is not the current generation of superhumans, capable of sequencing the entire human genome, proof of the efficacy of this policy?*. Où on constate: (1) que les phénomènes aléatoires de la procréation sont négligés : choisir un partenaire plutôt qu'un embryon dans un but eugénique, c'est faire abstraction des hasards génétiques qui président successivement à la production des gamètes (méiose) puis à leur rencontre (fécondation). Seule la sélection de l'œuf fécondé est digne d'un eugénisme scientifique, d'autant que des mutations, absentes chez les géniteurs, pourraient affecter le génome du nouvel être; (2) que l'auteur cède au vieux mythe d'une amélioration progressive de l'intelligence au cours de l'évolution humaine, postulat qui a nourri bien des fantasmes eugéniques. En fait, tout laisse croire que l'homme de Cro-Magnon était déjà doté de ces qualités de surhomme

\footnotetext{
* "Si une personne désire un enfant ayant certaines caractéristiques, mon conseil est de pratiquer l'eugénisme selon la mode actuelle, c'est-à-dire de se choisir un partenaire présentant les caractéristiques désirées. Après tout, la génération actuelle n'est-elle pas composée de surhommes capables de séquencer l'intégralité du génome humain, n'est-ce pas là la preuve de l'efficacité de cette politique? " (NDLR).
}

Figure 1. Le DPI du futur. Exemple de tri parmi 14 embryons de 4-6 cellules, 2 jours après FIV. L'élimination progressive des embryons "anormaux" (1), puis "à risque" (2) et de "second choix" (3), conduit à isoler ceux qui sont acceptables pour le transfert in utero (4). Pourquoi alors refuser au couple le choix du sexe, lequel est découvert à l'occasion du tri? 


\begin{tabular}{|c|c|}
\hline \multicolumn{2}{|c|}{$\begin{array}{c}\text { Tableau III } \\
\text { PROPOSITIONS POUR LIMITER LES DÉRIVES DU DPI }\end{array}$} \\
\hline Garde-fou proposé & Causes d'inefficacité \\
\hline Bonne pratique médicale & $\begin{array}{l}\text { Pression des patients } \\
\text { Pression de l'industrie } \\
\text { Enjeux économiques et de pouvoir }\end{array}$ \\
\hline Responsabilité des patients & $\begin{array}{l}\text { Crainte de la compétition sociale } \\
\text { pour l'enfant } \\
\text { Revendication d'un droit } \\
\text { au progrès médical }\end{array}$ \\
\hline $\begin{array}{l}\text { Liste limitative légale } \\
\text { des indications (1) }\end{array}$ & $\begin{array}{l}\text { Contradictoire avec la culture } \\
\text { (tolérance, dignité) } \\
\text { et avec les Droits de l'homme }\end{array}$ \\
\hline Agrément limité à 1 ou 2 centres & $\begin{array}{l}\text { Le label officiel permet d'éviter de } \\
\text { véritables contrôles } \\
\text { Risque de monopolisation }\end{array}$ \\
\hline Commission ad hoc a posteriori & $\begin{array}{l}\text { Peu d'effets sur les pratiques } \\
\text { Absence de critères de jugement } \\
\text { si pas de liste }\end{array}$ \\
\hline Commission ad hoc a priori & $\begin{array}{l}\text { Crédible seulement si: commision } \\
\text { élargie hors des professionnels, } \\
\text { avis motivé sur chaque dossier } \\
\text { avant DPI } \\
\text { Bilan annuel transparent et public }\end{array}$ \\
\hline
\end{tabular}

(1) Concernant le DPN, ces listes n'existent qu'au Japon et en Chine.

qui caractériseraient le généticien moderne...

L'opposition au DPI, telle que nous l'exprimons, est justifiée par l'absence de barrière au développement de cette technique (Tableau III) et donc la certitude qu'accepter aujourd'hui le DPI pour de bonnes raisons engage à le subir plus tard (dans 30 ans, dans 100 ans...) pour des raisons encore inexprimées. Aussi est-il dérisoire de défendre le DPI en soulignant ses limites actuelles, comme si elles devaient demeurer toujours et suffire à empêcher ses dérives.

Quand ils comparent les législations de plusieurs pays, Viville et al. semblent regretter les rigueurs de la loi française. En fait, les dispositions de la loi de 1994 sont remarquables en ceci qu'elles instituent de véritables limites au DPI puisqu'une seule "affection" peut être recherchée, et seulement après avis circonstancié d'un généticien du DPN. Soyons clairs: si ces dispositions avaient quelque chance d'être appliquées dans la transparence, nous ne saurions nous opposer à l'essor du DPI. démarche. Aussi, plutôt que d'inclure le DPI dans les progrès de la génétique médicale, nous y voyons la remise en cause de concepts fondamentaux qui fondent l'humanisme. Même si la technique est encore balbutiante, c'est dès aujourd'hui que l'humanité se trouve confrontée à cette remise en cause

\section{J. Testart}

Directeur de Recherche à l'Inserm, Unité Inserm U. 355, 32, rue des Carnets, 92140 Clamart, France.

\section{B. Sèle}

Professeur à la faculté de médecine de Grenoble, Inserm U. 309, Groupe de Biologie de la Reproduction, Faculté de Médecine, 38700 La Tronche, France.

\section{RÉFÉRENCES}

1. Viville J, Ray P, Wittemer C, Ohl J, Dellenbach $P$, Gerlinger $P$. Diagnostic génétique préimplantatoire : législation et aspects éthiques. medecine/sciences 1996; 12 : ???? 2. Testart J. Le désir du gène. Paris: Flammarion, 1994 .

3. Testart J, Sèle B. Towards an efficient medical eugenics: is the desirable always the feasible? Hum Reprod 1995; 10: 3086-90. 4. Veiga A, Santaló J, Vidal F, Caiderón G, Gimenez C, Boada M, Egozcue J, Barri P. Twin Gimenez C, Boada M, Egozcue J, Barri P. Twin
pregnancy after pre-implantation diagnosis for
sex selection. Hum Reprod 1994; $9: 2156-9$.

5. Avner P, Reubinoff B, Simon A, Zentner B-S, Friedmann A, Mitrani-Rosenbaum S, B-S, Friedmann A, Mitrani-Rosenbaum S, zation by preimplantation genetic diagnosis. Mol Hum Reprod 1996; 2 : 60-2.

6. Interview d'A. Handyside. J Int Med 1995; 360: $12-4$.

7. Fiddler M, Pergament E. Germline gene therapy: its time is near. Mol Hum Reprod 1996;2: 75-6.

8. Schulman J, Edwards RG. Preimplantation diagnosis is disease control, not eugenics. Hum Reprod 1996; 11: 463-4. 9. Geber S, Winston RM, Handyside A. Proli-
feration of blastomeres from biopsied cleaferation of blastomeres from biopsied clea-
vage stage human embryos in vitro: an alternative to blastocyst biopsy for preimplantation diagnosis. Hum Reprod 1995; 10: 1492-6.

10. Bongso A, Fong CY, Ng SC, Ratnam S. 10. Bongso A, Fong CY, Ng SC, Ratnam S.
Isolation and culture of inner cell mass cells from human blastocysts. Hum Reprod 1994; 9: $2110-7$.

1i. Handyside $\mathrm{AH}$. Commonsense as applied to eugenics: response to Testart applied to eugenics: response to T
and Sèle. Hum Reprod 1996; 11:707.

12. Fiddler M, Pergament E. Medically-assisted procreation: a maturing technology or a premature fear? Response to Testart and Sèle. Hum Reprod 1996;11: 708-9.

\section{TIRÉS À PART}

J. Testart. 